\title{
Pancytopenia and transient synovitis of hip joint in a SARS CoV-2 positive pregnant female: a case report
}

\author{
Amrita Gaurav $^{1 *}$, Juhi Mishra ${ }^{1}$, Rahul Sharma ${ }^{2}$, Kavita Khoiwal', \\ Rajkumar Yadav $^{2}$, Jaya Chaturvedi ${ }^{1}$
}

\begin{abstract}
${ }^{1}$ Department of Obstetrics and Gynaecology, ${ }^{2}$ Department of Physical Medicine and Rehabilitation, AIIMS, Rishikesh, Uttarakhand, India
\end{abstract}

Received: 19 May 2021

Accepted: 09 June 2021

\section{*Correspondence:}

Dr. Amrita Gaurav,

E-mail: aamrity@gmail.com

Copyright: ( ) the author(s), publisher and licensee Medip Academy. This is an open-access article distributed under the terms of the Creative Commons Attribution Non-Commercial License, which permits unrestricted non-commercial use, distribution, and reproduction in any medium, provided the original work is properly cited.

\section{ABSTRACT}

Pregnant women are at an increased risk for severe COVID-19 illness. Apart from the typical clinical manifestations, atypical presenting features of COVID-19 are also being found. We report the case of a 20 years old COVID positive antenatal patient with pancytopenia. The patient presented with scar tenderness and was taken up for emergency caesarean section at a platelet count of 5860 per microlitre. She was managed with intraoperative and postoperative transfusion of blood products. She developed chronic persistent hip pain and was diagnosed to have transient synovitis of the hip joint, which was managed conservatively. COVID-19 is a new disease with evolving clinical presentation. Pancytopenia and synovitis of hip are a rare manifestation of COVID-19 and has never been reported in a pregnant woman with COVID-19.

Keywords: Pancytopenia, Transient synovitis, Pregnancy, COVID-19

\section{INTRODUCTION}

On 30 January 2020, the World Health Organization (WHO) declared a public health emergency of international concern with the spread of COVID-19 disease and on 11 March 2020, COVID-19 pandemic was declared. Globally, as of February 2021, there have been $112,649,371$ confirmed cases of COVID-19, including 2,501,229 deaths. ${ }^{1}$ The first case reported in India was on 30 January 2020 in Kerala. Till now India has reported around 110 million cases, 108 million recovered and 150,000 deaths approximately. ${ }^{2}$

After witnessing more than a year with COVID-19, it is evident that patients with COVID-19 disease have shown varying clinical manifestation, ranging from asymptomatic infection to life threatening pneumonia and respiratory failure leading to death. ${ }^{3}$ Various areas are unexplored and clinical data is sparse regarding several aspects related to pregnant women. However, clinical experience of pregnancies complicated with COVID-19 infection suggests that pregnant woman should be considered potentially vulnerable to severe acute respiratory syndrome coronavirus-2 (SARS-CoV-2) infection. Physiological changes during pregnancy have a significant impact on the immune system, respiratory system, cardiovascular function and coagulation. Asymptomatic infection presents a further challenge regarding service provision, prevention and management. The impact of SARS-CoV-2 in pregnancy remains to be determined and a concerted, global effort is required to determine effects on implantation, fetal growth and development, labor and neonatal health. ${ }^{4,5}$

The involvement of almost every organ system has been reported in the patients with COVID-19 illness with varying incidences. The typical clinical features of COVID-19 are fever, dry cough, sore throat, dyspnea, fatigue, and myalgia. Less common presentations include headache, dizziness, nausea, abdominal pain, vomiting, 
and diarrhea. However, it has been reported that patients with COVID-19 might present with nonspecific or atypical symptoms such as hemoptysis, cardiac, neurological, gastrointestinal, ocular and cutaneous manifestations, as well as venous and arterial thrombosis which might delay testing, diagnosis and isolation. The exact pathophysiology behind these atypical symptoms is still not completely understood. ${ }^{6}$

The atypical presentations and routes of spread of COVID19 emphasize the importance of using appropriate personal protective equipment during every clinical contact with patients even after vaccination. Also, a high index of suspicion is required for early diagnosis of the patients for better management and also early isolation of suspected patients to limit the transmission of infection in the hospital and community settings.

\section{CASE REPORT}

We report the case of a 20 years old female at 35 weeks of gestation with previous lower segment caesarean section. Approval regarding reporting of this case was obtained from institutional review board and informed consent was obtained from the patient. Patient was diagnosed to be SARS CoV-2 15 days prior to admission at the present centre. After 15 days of testing positive, she visited the local health care centre with complaints of pain abdomen. Routine laboratory workup showed haemoglobin $6.3 \mathrm{~g} / \mathrm{dl}$, white blood cells (WBC) $3840 / \mathrm{cm}^{3}$ with $74 \%$ neutrophils and $25 \%$ lymphocytes and platelet count 30000 per microlitre. She was transfused with one unit packed red blood cells (RBC) and 2 units of random donor platelets (RDPs) and referred to the present centre which is a tertiary care referral centre in Uttarakhand, India for further management. Repeat laboratory workup at the present centre showed WBC $1110 / \mathrm{cm}^{3}$ with neutrophil $80.8 \%$ and lymphocytes $16 \%$, haemoglobin $8.1 \mathrm{~g} / \mathrm{dl}$ with a mean corpuscular volume of $85.8 \mathrm{fL}$, and platelet count was 5860 per microlitre. Her C-reactive protein (CRP) was $153.5 \mathrm{mg} / \mathrm{l}$ (normal range $0-5 \mathrm{mg} / \mathrm{l}$ ), D-dimer was $>5000 \mathrm{ng} / \mathrm{ml}$, lactate dehydrogenase (LDH) was $1564.7 \mathrm{U} / \mathrm{l}$ and serum ferritin was $306 \mathrm{ng} / \mathrm{ml}$. serum iron and vitamin B12 were normal. Folic acid deficiency was noted with serum Folic acid of $1.8 \mathrm{ng} / \mathrm{ml}$. Peripheral smear revealed dimorphic anaemia. SARS-CoV-2 real time-polymerase chain reaction (RT-PCR) was persistently positive.

On general examination, the patient had mild pallor and tachycardia with pulse of 130 beats per minute (bpm) regular. On abdominal examination, she was corresponding to 32 weeks period of gestation and was having 2-3 contractions in 10 minutes each lasting for 2530 seconds. Scar tenderness was elicited on palpation. The patient was taken for lower segment caesarean section under general anaesthesia. 2 units of packed RBCs, 4 units of RDPs and 4 units of FFP were transfused intraoperatively. A live $2.15 \mathrm{~kg}$ female baby was delivered. Intraoperative and Postoperative period was uneventful. 3 units PRBCs and 10 units of RDPs were transfused in the immediate postoperative period. During the second week of her admission, the platelet count showed a recovery but the patient developed fever. There was no evidence of puerperal sepsis or breast engorgement. procalcitonin was raised with the value of $31.79 \mathrm{ng} / \mathrm{ml}$. IgM for dengue, malaria and typhoid along with urine and blood cultures were negative. A repeat test for COVID-19 was done on 30th day of her first positive test which was still positive. She also complained of pain in the back of right thigh and developed a limp while walking. Ultrasound Doppler was performed for screening of deep vein thrombosis was ruled out by Doppler ultrasound. The patient was examined by the team from physical medicine and rehabilitation. On examination patient was lying down in bed with right hip joint in an attitude of flexion, abduction and external rotation. Patient was unable to complete full range of motion of the affected joint secondary to pain. Both internal and external rotation were markedly restricted and flexion, abduction and external rotation test (FABER) positive on the affected side. Sacro iliac joints were found to be unaffected. Patient was walking with antalgic gait with reduced stance phase on right side with hip in slight flexion, abduction and external rotation. Considering the history of fever along with examination findings Transient synovitis and septic arthritis of hip were kept as working diagnoses. $\mathrm{X}$ ray showed no obvious joint changes (Figure 1). Thus magnetic resonance imaging (MRI) of the hip joint was done which showed hyperintense marrow signal with joint effusion and soft tissue oedema consistent with transient synovitis of hip. Patient was advised to avoid weight bearing and skin traction was applied. Meanwhile, the pancytopenia resolved in the repeat laboratory tests with only moderate anaemia remaining. The bone marrow examination and synovial fluid examination was not performed as it was in its resolving phase and patient had financial constraints.

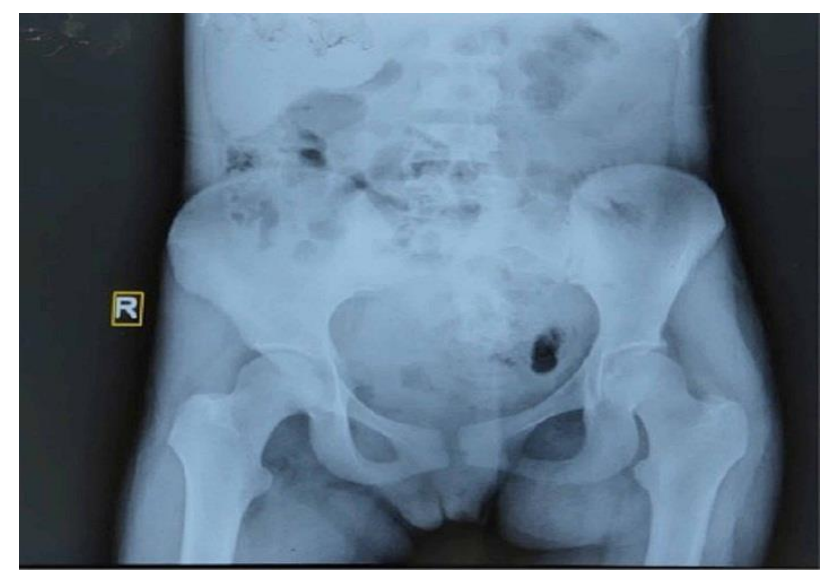

Figure 1: X-ray showing bilateral hip joints.

Patient was discharged on $21^{\text {st }}$ postoperative day of LSCS along with the healthy baby. She was afebrile and asymptomatic upon discharge. WBC and platelet counts had recovered, but she still had moderate anaemia which 
was managed by oral haematinics. Her pain in the hip joint was resolved and she was discharged with the skin traction applied.

The final clinical impresson was of a SARS CoV-2 positive postnatal female with persistent viraemia with pancytopenia (treated) with synovitis of the hip joint.

\section{DISCUSSION}

Pregnant women are considered to be at an increased risk for severe COVID-19 illness and preventive measures of COVID-19 should be emphasized for them. Awareness amongst the women about their potential risk is needed to reduce severe COVID-19-associated illness. ${ }^{4,5}$ A review by Abobaker et al emphasized on atypical presentations of COVID 19. They found haemoptysis, pulmonary embolism, cardiac manifestations, neurological manifestations, gastrointestinal, ocular, cutaneous and male reproductive health disorders as the major atypical presentations. $^{6}$

The present case scenario consists of a pregnant COVID19 patient with involvement of the haematological system in the form of pancytopenia and musculoskeletal system in the form of transient synovitis of the hip joint.

The pattern of haematological involvement in COVID-19 patients has not been well-established in literature, but few cases of pancytopenia have been reported where bone marrow biopsy showed nonspecific reactive changes. Bone marrow suppression is a well-established symptom of other viral infections and has been seen in Epstein-Barr virus (EBV), human-immunodeficiency virus (HIV), cytomegalovirus (CMV), and parvovirus B19 infection. ${ }^{7}$

"Cytokine storm" which has been found to contribute to mortality in COVID-19 cases is a state of high concentration of pro-inflammatory cytokines which affect hematopoietic stem cells and impair haematopoiesis. Also, bone marrow has angiotensin-converting enzyme 2 receptor which acts as a target of the SARS-CoV-2. ${ }^{8}$ Also, recently, it has been found that lung is a site for platelet biogenesis and a reservoir for hematopoietic progenitors. So, with the lung injury caused by SARS-CoV-2 infection, lung hematopoietic progenitors are destroyed, thus contributing to the pancytopenia. ${ }^{9}$

Issa et al reported the first COVID-19 case of pancytopenia in an immunocompromised 53-year-old man who was in intensive care unit for acute respiratory distress and cytokine release syndrome. As the haematological involvement was a not well established, blood and bone marrow aspiration was performed to establish the diagnosis. SARS-CoV-2 reverse transcription-PCR (RTPCR) was negative in nasopharyngeal swab samples, but had positive findings in bronchoalveolar lavage fluid, blood and bone marrow aspiration samples. No other viruses were detected. ${ }^{10}$
The other reported case of pancytopenia by Hersby et al was a known case of follicular lymphoma characterized by a much milder clinical course and COVID-19 was not detected in the bone marrow aspirate. The cytopenia resolved after the patient came negative for SARS-CoV$2 .^{11}$ Further, cases and literature are needed for establishment of a correlation and definitive diagnostic and management criteria.

Although there is no established causal relationship between pregnancy and pancytopenia, but, pancytopenia affects the feto-maternal outcome. The major maternal risks are antepartum and postpartum haemorrhage, sepsis, cardiac failure, and preterm labor. The fetal risks include fetal growth restriction, fetal thrombocytopenia and intrauterine fetal demise. ${ }^{12}$

Severe thrombocytopenia is a serious concern and a minimum platelet count of 50,000/ul is considered optimal for any surgery. General anaesthesia is preferred over regional as there is a risk of spinal hematoma formation in patients with severe thrombocytopenia. ${ }^{13}$ However, in the present case this optimal level of platelet count could not be achieved because of the emergency situation of scar dehiscence. Hence, an adequate supportive therapy and transfusions were given during the intra-operative and post-operative period with an aim to maintain maternal $\mathrm{Hb}$ of $8 \mathrm{~g} / \mathrm{dl}$ and the platelet count of 20,000/ul as per the British society for haematology guideline. ${ }^{14}$

Association of viral arthralgia has been reported in $14.9 \%$ of cases but data on rheumatic and inflammatory manifestations (such as arthritis) are scarce and its association with SARS-CoV-2 has not yet been established. Studies have suggested that $1 \%$ of the cases with acute arthritis had viral etiology, most commonly, parvovirus B-19, hepatitis B and C. HIV and the alpha viruses. ${ }^{15}$ Sinaei et al reported two paediatric COVID-19 patients who developed limping and were diagnosed to have synovitis of the hip joint. Both these patients were managed conservatively using NSAIDs and responded to management. ${ }^{16}$ Parisi et al reported a case where a 58 year old woman developed an ankle arthritis 25 days after being tested positive for SARS-CoV-2 with slight increase in Creactive protein. She had non severe symptoms and was managed conservatively by NSAIDs. ${ }^{17}$

There is paucity of literature on rheumatological manifestations of COVID-19 and further reported cases will help clarify its pathogenicity in the joints.

\section{CONCLUSION}

To the best of our best knowledge, the present case is the third report of rheumatological manifestation of COVID19 and the first such case of pancytopenia and synovitis of the hip joint in a pregnant woman. We report this case to contribute to the existing literature for further research in determining the preventive and curative measures. 
Funding: No funding sources

Conflict of interest: None declared

Ethical approval: Not required

\section{REFERENCES}

1. WHO Coronavirus Disease (COVID-19) Dashboard. (2020). Available at: https://covid19.who.int. Accessed on 22 March 2020.

2. Ministry of Health and Family Welfare, GOI. Available at: https://mohfw.gov.in. Accessed on 15 March 2021.

3. Chow A, Htun H, Kyaw W. Atypical COVID- 19: Preventing transmission from unexpected cases. Infect Control Hospital Epidemiol. 2020;1-3.

4. Khoiwal K, Kapur D, Gaurav A. Management of Pregnant Women in Times of Covid- 19: A Review of Current Literature. J Obstet Gynecol India. 2020;70:262-6.

5. Wastnedge E, Reynolds R, van Boeckel S, Stock S, Denison F, Maybin J, Critchley H. Pregnancy and COVID-19. Physiol Rev. 2021;101:303-18.

6. Abobaker A, Raba AA, Alzwi A: Extrapulmonary and atypical clinical presentations of COVID-19. J Med Virol. 2020;92:2458-64.

7. Pascutti MF, Erkelens MN, Nolte MA. Impact of Viral Infections on Hematopoiesis: From Beneficial to Detrimental Effects on Bone Marrow Output. Front Immunol. 2016;16:364-410.

8. Li MY, Li L, Zhang Y, Wang XS. Expression of the SARS-CoV-2 cell receptor gene ACE2 in a wide variety of human tissues. Infect Dis Poverty. 2020;28:45-10.

9. Lefrançais E, Ortiz-Muñoz G, Caudrillier A. The lung is a site of platelet biogenesis and a reservoir for haematopoietic progenitors. Nature. 2017;6:105-9.
10. Issa N, Lacassin F, Camou F. First case of persistent pancytopenia associated with SARS- CoV-2 bone marrow infiltration in an immunocompromised patient. Ann Oncol. 2020;31:1418-9.

11. Hersby DS, Do TH, Gang AO, Nielsen TH. COVID19-associated pancytopenia can be self- limiting and does not necessarily warrant bone marrow biopsy for the purposes of SARS- CoV-2 diagnostics. Ann Oncol. 2021;32:121-3.

12. Deka D, Malhotra N, Sinha A, Banerjee N, Kashyap R, Roy KK. Pregnancy associated aplastic anemia: Maternal and fetal outcome: Pregnancy associated with aplastic anemia. J Obstet Gynaecol Res. 2003;29:67-77.

13. Rathore S, Pramanick A, Regi A, Lionel J. Aplastic anemia in pregnancy. J Obstet Gynaecol. 2014;64:268.

14. British Committee for Standards in Haematology, Blood Transfusion Task Force: Guidelines for the use of platelet transfusions. Br J Haematol. 2003;122:1023.

15. Marks M, Marks JL. Viral arthritis. Clin Med. 2016;16:129-34.

16. Sinaei R, Pezeshki S, Sinaei R. Post nCoV-2 limping Child: Report of two cases and a rapid Review. In Review.

17. Parisi S, Borrelli R, Bianchi S, Fusaro E. Viral arthritis and COVID-19. Lancet Rheumatol. 2020;2:655-7.

Cite this article as: Gaurav A, Mishra J, Sharma R, Khoiwal K, Yadav R, Chaturvedi J. Pancytopenia and transient synovitis of hip joint in a SARS CoV-2 positive pregnant female: a case report. Int J Reprod Contracept Obstet Gynecol 2021;10:2910-3. 